\title{
Research on Electromechanical Four-Point Leveling Control System Based on MPC
}

\author{
Shuailin Li, Yi Zhou, Deming Ou, Wen Wang \\ School of Mechanical Engineering, Chongqing University 400044
}

\begin{abstract}
Multi-supporting leveling platform was wildly applied in areas of normal or military industry. To complete some frocking targets, it usually requires good performance of levelness and short time adjustment. It is very meaningful to enhance the automatic level of leveling platform by mining adaptive controlling algorithm which satisfied the levelling error and used shorter time. In this paper, four-point supporting electromechanical leveling system was set as the object to study. By analyzing the leveling characteristic of the system, appropriate leveling strategies were put forward. The simulation result of the system, whose model was built based on MPC, show that this way achieves good auto leveling performance.
\end{abstract}

Keywords: Four-Point Supporting; Leveling; Position Error; MPC

\section{Introduction}

At present, civil industrial fields such as vertical shaft construction, high-altitude rescue, road construction and other aspects, as well as some military confrontation processes. Such as missile artillery launching, radar searching for enemies and other links, especially in the cutting-edge areas of national defense and military industry, its tooling equipment or load. Platforms often have levelness requirements during operation ${ }^{[1,2]}$ and some even have leveling speed and accuracy requirements. There are various types, divided into three support leveling, four support leveling and six support leveling according to the support structure, divided into driving modes. There are two types of organic electro-mechanical type and electro-hydraulic type. Among them, the four-point support leveling platform has excellent anti-overturning capability, good rigidity and leveling. The relative complexity is relatively low and stands out. Electro-hydraulic leveling system often has non-linearity, slow response, oil leakage and other phenomena. At the same time, it is slightly inferior to electromechanical leveling system in cost, stability and maintainability.

The self-leveling system itself is a complex time-varying nonlinear system. In order to achieve faster leveling speed and higher adjustment, flat precision and high stability, in the selection of control strategy to choose more appropriate, specifically or control algorithm, such as classical PID control algorithm. As the performance requirements of leveling system become higher and higher, in order to meet the more complex. The control system requires more practical methods. Model Predictive Control algorithm. MPC is an effective control method developed in the process of industrial practice, which adopts a non-minimum description model. Robustness and stability are good, the precision requirement of the model is not high, the modeling is convenient, meanwhile, a rolling optimization strategy is adopted. Good dynamic performance, ${ }^{[4-6]}$ and its char-

\footnotetext{
Copyright (C) 2020 Shuaili Li et al.

doi: 10.18282/fme.v2i1.840

This is an open-access article distributed under the terms of the Creative Commons Attribution Non-Commercial License

(http://creativecommons.org/licenses/by-nc/4.0/), which permits non-commercial use, distribution, and reproduction in any medium, provided the original work is properly cited.
} 
acteristics are in line with the control requirements of the automatic leveling system.

This paper puts forward and analyzes the leveling characteristics of an electromechanical four-point leveling system, selects the appropriate leveling strategy, adopts the base. The control algorithms of Yu and MPC have been simulated for leveling system. The leveling speed and leveling accuracy meet the system requirements well, which proves the correctness and applicability of predictive control method in automatic leveling system.

\section{Leveling system and control strategy}

The bottom of each support leg of the leveling platform is fixed on the horizontal ground (frame) and is driven by a vertically mounted motor-ball screw. A dual-axis inclinometer placed in the center of the platform measures the angle of the platform at this time and feeds back the result to the control system, which is roughly adjusted and finely adjusted. After that, the platform gradually tends to a horizontal state, and the actuator stops working and locks to keep the state of the system unchanged until the next time is given. Leveling instruction or withdrawal instruction. The control strategy of the four-point leveling system can be roughly divided into angle error control and position error control. Angle error control is specific. In order to take the inclination angle of the vehicle body platform in two horizontal directions detected by the angle sensor as a reference, the height of each leg is adjusted to be straight. Until these two inclination values are reduced to within the set accuracy. This method bypasses the complicated decoupling calculation, but adjusts independently.

One direction will affect the levelness of the other direction, requiring repeated adjustment and taking a long time. The position error control method can be divided into (1) aligning with the highest point; (2) aligning with the lowest point; (3) aligning with the center. ${ }^{[7]}$ According to the Z-direction height of the four support points in the reference system when the platform is in the initial state. Taking the leveling strategy of aligning with the highest point and aligning with the highest point as an example, its concrete implementation is as follows: the controller judges the height of the four support points according to the inclination signals collected by the two-axis inclination sensors, and calculates the height errors of the remaining 3 support points and the highest support point. The error value is the displacement amount of the support leg rising. After the appropriate control algorithm is selected and calculated, it is sent to the input end of the servo system, driving each support leg to rise to the target position, and leveling is finished.

Since the leveling platform is fixed at the bottom, the mode of lifting and adjusting the top platform is adopted, meanwhile, the working efficiency and elimination are considered. In addition to the influence of reverse clearance and dead zone of servo drive system, in order to improve the accuracy and shorten the leveling time as much as possible, the height error control and leveling strategy of aligning points is finally adopted.

\section{Predictive control model of leveling system}

The self-leveling system is regarded as a pure rigid system, and the initial and final states of the system can be observed. So, the model of the system is simplified and expressed as a pure geometric relation model with two states (initial and final). The platform is $a$ in length, $b$ in width and $12^{\prime} 3^{\prime} 4$ ' in surface. 


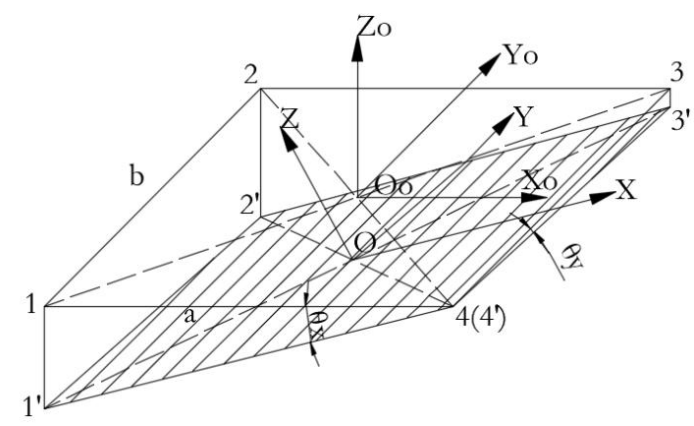

Figure 1. Inclined state diagram of rigid platform.

When the platform has a small horizontal inclination angle, it can be considered to be mainly caused by the geometric unevenness of the platform. The large horizontal inclination angle is mainly caused by the geometric unevenness of the platform and the rotation angle generated by the deformation of the platform, and the larger the inclination angle is the larger the deformation of the platform is. According to the selected leveling method, it can be considered that the longitudinal inclination angle $\mathrm{x}(\mathrm{t})$ and the longitudinal rotation angle $\mathrm{y}(\mathrm{t})$ satisfy some tangent relation, as shown in Figure 3.

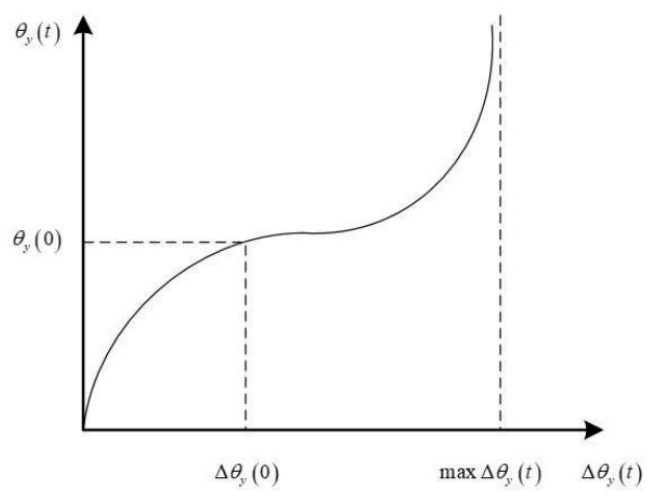

Figure 3. Variation relationship of vertical inclination angle of platform.

\section{Simulation test analysis}

In order to verify the effectiveness of the MPC algorithm proposed in this paper in the application of the automatic leveling system, the Matlab/Simulink is used to simulate and analyze the rigid model of the automatic leveling control system, and the iteration period of simulation prediction is taken. According to the actual engineering needs, the leveling time is determined, leveling error and the simulation parameters are shown in the following Table.

\begin{tabular}{cccc}
\hline$Q_{x}$ & $\pi / 2$ & $Q_{y}$ & $\pi / 2$ \\
$k_{x}$ & $1 / 2 \pi$ & $k_{y}$ & $1 / 2 \pi$ \\
$a$ & $1400 \mathrm{~mm}$ & $b$ & $800 \mathrm{~mm}$ \\
$\theta_{x}(0)$ & $3^{\circ}$ & $\theta_{y}(0)$ & $-1.8^{\circ}$ \\
\hline
\end{tabular}

Table 1. Simulation parameters

In the following simulation results, Figure 4 is the horizontal angle change curve of the platform during the simulation leveling process, and Figure 5 is the vertical horizontal angle change curve of the platform during the simulation leveling process. 


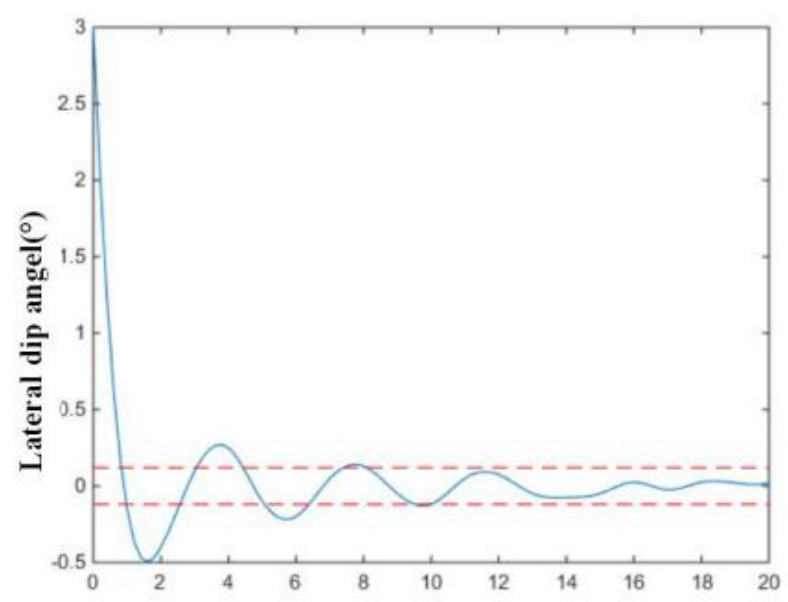

Figure 4. Simulation curve of later Angle change.

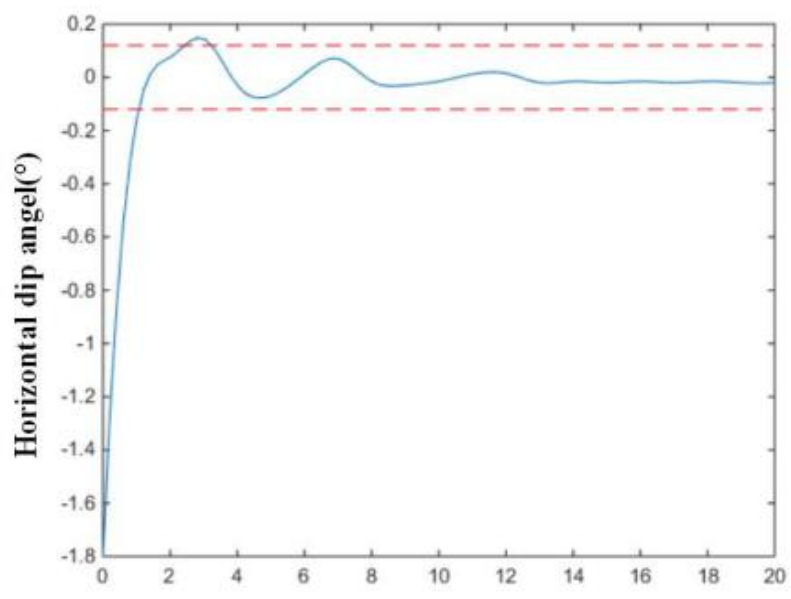

Figure 5. Simulation curve of longitudinal angle change.

Under the condition of leveling decoupling, it can be seen that the rigid model predictive control leveling speed is satisfactory. As can be seen from Figures 4 and 5, after about 12 times of prediction iteration adjustment, the lateral inclination angle and the longitudinal inclination angle both converge to the accuracy range required for leveling. At this time, the leveling time requirement is met.

\section{Conclusion}

This paper studies the leveling strategy of the electromechanical four-support automatic leveling system, adopts the algorithm based on MPC to establish the prediction model and state equation of the leveling system, and makes simulation analysis. In the whole leveling simulation test, the leveling system has reached the precision requirement, and the platform horizontal angle overshoot is small, the leveling response is fast, and good leveling effect has been achieved. Therefore, the predictive control method based on state equation can be considered for future automatic leveling systems.

\section{References}

1. Ling Xuan, Wang Xudong, Chen Seke, et al. Simulation study on synchronous control of hydraulic lifting system of radar antenna vehicle. Machine Tool and Hydraulic 2013; 41(8): 81-83.

2. Li Degang. Research on synchronous control of hydraulic leveling system based on fuzzy PID. China New Communications 2017; 19(3): 148-149.

3. $\mathrm{Li}$ Guangwei, $\mathrm{Xu}$ Xinfang. Electromechanical automatic leveling system. Military Automation 2013; 32(3): 1-2.

4. Zhang Shihan, Liu Zhenjian, Qiu Jinbo. Application of model predictive control model in shearer height adjustment system. Industrial Automation 2018; 44(5): 42-46.

5. Devin W Griffith, Sachin C Patwardhan, Lorenz T Biegler. Robustly stable Adaptive horizon nonlinear model predictive control. Journal of Process Control 2018; 70(138): 109-122.

6. Liu Zhe, Song Rui, Zou Tao. Tracking control algorithm for end force of grinding robot based on model predictive control. Journal of Shandong 
University (Engineering Edition) 2018; 48(1): 42-49.

7. Zhao Jingyi, Yang Yujing, Kang Shaopeng, et al. Research and application of four-point support "face-chasing" leveling strategy for self-propelled hydraulic flat cars. Machine Bed and Hydraulic
Pressure 2015; 43(15): 57-60.

8. Shu Yahai, Zhuang Wenxu, Li Kexiang, et al. Virtual leg compensation control strategy of a four-point electromechanical leveling system and electromechanical joint simulation analysis. Radar and Confrontation 2017; 37(1): 55-59. 